\title{
SOME SEMIGROUPS ON THE TWO-CELL
}

\section{ANNE LESTER ${ }^{1}$}

A topological semigroup is a nonvoid, Hausdorff space, $S$, together with a continuous, associative multiplication defined on $S$. Here the term semigroup will always denote a topological semigroup. In [4], Wallace and Koch have shown that if the circle, $B$, is a semigroup such that $B^{2}=B$, then either $B$ is a group, or the multiplication on $B$ is of the trivial kind $x y=x$, or $x y=y$. In [6], Mostert and Shields give a description of a semigroup on the two-cell where the boundary of the two-cell relative to the plane is a group. The main results of this paper will be a description of a semigroup on the two-cell where the multiplication satisfies the following two conditions: one; for $x$ and $y$ in the boundary of the two-cell relative to the plane $x y=x$, and two; there is a zero but no other idempotent in the interior of the two-cell. The following theorem will be proved:

THEOREM A. Let $S$, topologically the two-cell, be a semigroup with zero in the interior. Assume for $x$ and $y$ in $B$, the boundary of $S$ relative to the plane, $x y=x$. Suppose, moreover, that $S$ has no idempotents, except zero, in the interior. Then there exists an I-semigroup $T$ in $S$ such that $S=B T$. Also for $e$ and $f$ in $B, t$ and $s$ in $T(e s)(f t)=e(s t)$; and if $e s=f t$ then $s=t$.

There is, as will be obvious from the proof, a left-right dual of this theorem. Due to the length of the proof of this theorem, which will be given in a sequence of lemmas and theorems, some definitions and background material have been omitted. The reader is referred to [6] for any definitions and results not given here.

I wish to thank Dr. P.S. Mostert for his patient and understanding help. It was he who suggested this problem and supplied the material for Theorem 1. I also wish to thank Prof. A. D. Wallace for his encouragement and help.

A semigroup $P$ will be said to satisfy condition $(\alpha)$ if (i) there exists a set $A$ in $P$ such that $A \subseteq f(A)$, where $f$ is the function defined by $f(x)=x^{2}$ for all $x$ in $P$; and (ii) there exists $y$ in $A$ such that $y \neq y^{2}$. In the following discussion $P$ will be assumed to be a compact semigroup which satisfies condition $(\alpha)$. For an element $p$ in $A$ such that

Presented to the Society, August 28, 1958; received by the editors September 26, 1958.

${ }^{1}$ Based on a thesis submitted in partial fulfillment of the requirements for the Master of Science degree at Tulane University. 
$p \neq p^{2}, s(p)$ will denote a fixed sequence of elements $p_{n}, n=0,1,2, \ldots$ with the properties that (i) $p_{0}=p$, and (ii) $p_{n}^{2}=p_{n-1}$. For positive integers $n$ and $j$, let $Z_{n, j}(s(p))$ be defined as follows:

$$
Z_{n, j}(s(p))=\left\{p_{m}^{i}: \frac{i}{2^{m}} \leqq \frac{j}{2^{n}} \text { and } p_{m} \in s(p)\right\}^{*},
$$

where $A^{*}$ denotes the topological closure of a set $A$. For real, nonnegative $t$, let $Z_{t}(s(p))=\bigcap\left\{Z_{n, j}(s(p)): j / 2^{n}>t\right\}$. Also let $\delta\left(Z_{t}(s(p))\right.$ $=Z_{t}(s(p)) \backslash \bigcup_{r<t} Z_{r}(s(p))$, where $A \backslash B$ denotes the set theoretic difference of $A$ by $B$. In the following discussion consider the element $p$ in $A$ and the sequence $s(p)$ fixed and let $Z_{0}(s(p))$ be denoted by $Z$.

Lemma 1. $Z=\bigcap_{t>0}\left\{Z_{t}(s(p))\right\}$ and $Z$ is a compact, commutative group.

Proof. Clearly $Z=\bigcap_{t>0}\left\{Z_{t}(s(p))\right\}$ and $Z$ is nonvoid since $P$ is compact and $Z$ is the intersection of a tower of closed subsets of $P$. Since $Z$ is closed it is also compact. Using the fact that multiplication is continuous in $P$ and that the sequence $s(p)$ was chosen so that $p_{n}^{2}=p_{n-1}$, it can be easily shown that $Z$ is a commutative subsemigroup of $P$ and for $a$ in $Z, p_{n}$ in $s(p)$ that $p_{n} \in a P \cap P a$. From this last fact it follows that any idempotent in $Z$ acts as an identity for the sequence $s(p)$, and therefore for all of $Z$. Since $Z$ is a compact subsemigroup of $P$ it contains at least one idempotent. But any idempotent in $Z$ acts as an identity, hence $Z$ can contain at most one idempotent. Thus $Z$ is a group, [9].

LeMmA 2. If $y$ and $z$ belong to $\delta\left(Z_{t}(s(p))\right)$, there exists $g$ in $Z$ such that $y=g z$.

Proof. Since $y$ and $z$ are in $\delta\left(Z_{t}(s(p))\right), y=\lim p_{m_{n}}^{i_{n}}$ and $z=\lim p_{\boldsymbol{k}_{n}}^{j_{n}}$ where $p_{m_{n}}$ and $p_{k_{n}}$ are subsequences of $s(p)$ and $i_{n} / 2^{m_{n}} \rightarrow t^{+} \leftarrow j_{n} / 2^{k_{n}}$. We may assume that $i_{n} / 2^{m_{n}} \geqq j_{n} / 2^{k_{n}}$ for all $n$. Let $r_{n} / 2^{a_{n}}=i_{n} / 2^{m_{n}}$ $-j_{n} / 2^{k_{n}}$. Now $r_{n} / 2^{q_{n}}$ converges to zero, therefore $\left\{p_{q_{n}}^{r_{n}}\right\}$ clusters in $Z$. Since a subsequence of $\left\{p_{q_{n}}^{r_{n}}\right\}$ converges to some $g$ in $Z$, let us assume that the sequence converges to $g$. Hence we have, $y=\lim p_{m_{n}}^{i_{n}}$ $=\left(\lim p_{q_{n}}^{r_{n}}\right)\left(\lim p_{k_{n}}^{j_{n}}\right)=g z$.

TheORem 1. Let $P$ be a compact semigroup satisfying condition $(\alpha)$. For $p$ in $A$ such that $p \neq p^{2}$ let $Z(s(p))$ be defined as above. Then there exists a one-parameter semigroup $\sigma$ from $[0,1]$ to $Z_{1}(s(p))$, such that $\sigma(0)=e$, the identity of $Z(s(p))$, and $\sigma(1) g=p$ for some $g$ in $Z(s(p))$.

Proof. The proof of this theorem is long and is divided into a sequence of short steps. 
(i) Since $e \in Z(s(p))$, there is a sequence $\left\{p_{m_{n}}^{i_{n}}\right\}$ such that $e=\lim p_{m_{n}}^{h_{n}}$ and $i_{n} / 2^{m_{n}}$ converges to zero. For notational reasons, let $r_{n}=i_{n} / 2^{m_{n}}$ and $p_{m_{n}}^{i_{n}}=p^{r_{n}}$. For each $n$, define $\theta(n)=\left[1 / r_{n}\right]$, where $[a]$ is the least integer greater than or equal to $a$. From the definition of $\theta(n)$ and the fact that $r_{n} \rightarrow 0$, it follows that $\theta(n) r_{n} \rightarrow 1^{+}$.

For each real number $s$, such that $0<s \leqq 1$, define a function $f_{s}$ from $J$, the positive integers, to $Z_{1}(s(p))$ by $f_{s}(n)=p^{[s \theta(n)] r_{n}}$. For each $s, f_{s}$ is a continuous function and therefore has a unique extension, $f_{s}^{*}$, to $\beta(J)$, the Stone-Cech compactification of $J$. Let $\alpha \in \beta(J) \backslash J$ be chosen and considered fixed in the following discussion.

For each real $s, 0<s \leqq 1$, define $\tau(s)=f_{s}{ }^{*}(\alpha)$ and $\tau(0)=e$. From the definition of $\tau$ it can be shown that for $s, t$ and $s+t$ in $[0,1], \tau(s+t)$ $=\tau(s) \tau(t)$. Also from the definition of $\tau$ and the fact that $[\theta(n) t] r_{n} \rightarrow t^{+}$, it follows that $\tau(t) \in Z_{t}(s(p))$ and $\tau(t)$ does not belong to $Z_{r}(s(p))$ for any $r<t$, that is, $\tau(t) \in \delta\left(Z_{t}(s(p))\right)$ for $0<t \leqq 1$.

(ii) $G$, defined by $G=\bigcap_{t>0}\left\{(\tau[0, t])^{*}\right\}$, is a group with identity $e=\tau(0)$. Clearly $e \in G$ and $G \subseteq Z(s(p))$. Thus it remains to show that $G^{2} \subseteq G$, since $G$ is compact and contains only one idempotent which acts as an identity. By a straightforward argument using the facts that multiplication is continuous in $P$ and that for $s, t$ and $s+t$ in $[0,1], \tau(s+t)=\tau(s) \tau(t)$, it follows immediately that $G^{2} \subseteq G$.

(iii) Let $Q$ be defined by $Q=(\tau[0,1 / 2))^{*}$. $Q$ is a compact, adequate local semigroup with maximal subgroup $G$. This statement follows almost immediately from the facts that if $x$ is in $Q$ then $x=\tau(a) g$ for some $a$ such that $0 \leqq a \leqq 1 / 2$, and some $g \in G$. To show the latter let us assume that $x$ belongs to $Q$. Then there is a sequence $a_{n}$ in $[0,1 / 2]$ such that $x=\lim \tau\left(a_{n}\right)$. Since $a_{n} \in[0,1 / 2)$ for each $n$, there is a real number $t$ in $[0,1 / 2]$ such that $t=\lim a_{m}$, where $a_{m}=a_{n_{j}}$ is some subsequence of $a_{n}$. We may assume that $a_{m} \leqq t$ for each $m$, or $a_{m} \geqq t$ for each $m$. If $a_{m} \leqq t$, let $b_{m}=t-a_{m}$. Then $\tau(t)=\tau\left(a_{m}\right) \tau\left(b_{m}\right)$ and $b_{m} \rightarrow 0$. Hence $\tau(t)=\lim \tau\left(a_{m}\right) \lim \tau\left(b_{m}\right)$. That is $\tau(t)=x g$ for some $g \in G$. Thus $x=\tau(t) g^{-1}$. The case for $a_{m} \geqq t$, for each $m$, is similar.

Now suppose $x$ and $y$ are in $Q$ and $x y=e$. By the preceding argument $x=\tau(a) g$ and $y=\tau(b) h$ for some $a$ and $b$ in $[0,1 / 2], g$ and $h$ in $G$. Since $x y=e$, by (ii) we may conclude that $a+b=0$, hence $a=b=0$. Thus $x=g$ and $y=h$ and both belong to $G$. This concludes the proof of the above statement.

It is not difficult to show that $Q$ contains no idempotents except $\tau(0)$, hence by [5], there is a one-parameter semigroup $\gamma$ from $[0,1]$ to $Q$ such that $\gamma(t) \in G$ if and only if $t=0$. Reparametrize $\gamma$ so that $\gamma(t) / G=\tau(t) / G$ and denote the new one-parameter semigroup by $\sigma$. This gives the first part of Theorem 1. To obtain $p=\sigma(1) g$ for some 
$g$ in $Z(s(p))$, we need only to note that $p$ and $\sigma(1)$ both belong to $\delta\left(Z_{1}(s(p))\right)$ and apply Lemma 2 .

Proof of Theorem A. Throughout this proof $S$ will denote a semigroup satisfying the hypothesis of Theorem A.

Lemma 3. For each $a$ in $S$, there exists an $x$ in $S$ such that $x^{2}=a$.

Proof. Define $f: S \rightarrow S$ by $f(x)=x^{2} . f$ is a continuous function and for each $e$ in $B$, the boundary of $S, f(e)=e^{2}=e$. If $f$ does not map $S$ onto $S$, then it would be possible to retract $S$ onto $B$ which presents a contradiction. Thus $f$ maps $S$ onto $S$ and the lemma is proved.

Let $p \neq 0$ be in $S^{0}$, the interior of $S$, and let $s(p)$ be a fixed sequence of $2^{n}$ th roots of $p$ as defined above. Let $e$ denote the identity of $Z(s(p))$, which, by the hypothesis on $S$, belongs to $B$. Denote by $\sigma$ the one-parameter semigroup as constructed in Theorem 1 such that $\sigma(0)=e$.

Lemma 4. For $t$ in $(1, \infty)$, define $\tau(t)=\sigma(1)^{n} \sigma(a)$, where $t=n+a, n$ an integer and $0 \leqq a<1$. For $t$ in $[0,1]$ define $\tau(t)=\sigma(t)$. $\tau$ so defined is a continuous homomorphism from $R$, the additive semigroup of reals $\geqq 0$, into $S$ and $(\tau[0, \infty))^{*}$ is a compact, connected, commutative subsemigroup with identity $e$.

Proof. The proof of this lemma makes frequent use of the fact that $\sigma(1)=\sigma(t) \sigma(1-t)$ for any $t$ in $[0,1]$. Using this it is easy to see that $\sigma(1)^{n} \sigma(t)=\sigma(t) \sigma(1)^{n}$ and hence from the definition of $\tau$, that $\tau(a+b)$ $=\tau(a) \tau(b)$ for all real $a$ and $b$. This proves that $\tau$ is a homomorphism from $R$ into $S$. Since $\tau[0, \infty)$ is a commutative, connected subsemigroup of $S$, it follows that $(\tau[0, \infty))^{*}$ is again one such.

In the following lemma, $J(x)=x \cup x S \cup S x \cup S x S, \quad J_{x}$ $=\{y: J(y)=J(x)\}$ and $I(x)=J(x) \backslash J_{x}$.

Lemma 5. Let $C$ be a continuum that is a semigroup with zero. Let $A=A^{*}$ be a subset of $C$ such that for some $x_{0}$ in $C$ the following holds: (1) $A^{*} \backslash A^{0} \subseteq J_{x_{0}}$; (2) $J_{x_{0}} \neq\left\{x_{0}\right\}$; and (3) $A^{0}$ is nonvoid and is contained in $J\left(x_{0}\right)$. Then the zero for $C$ belongs to $A^{0}$.

Proof. In [9], Wallace proves that if $C$ is a continuum and $J_{x_{0}} \neq\left\{x_{0}\right\}$ then $I\left(x_{0}\right)^{*}=J\left(x_{0}\right)$ and $I\left(x_{0}\right)$ is connected. By the above hypothesis on $A$ we have $I\left(x_{0}\right) \cap A=\left(I\left(x_{0}\right) \cap A^{0}\right) \cup\left(I\left(x_{0}\right) \cap A^{*} \backslash A^{0}\right)$ $=I\left(x_{0}\right) \cap A^{0}$. This implies that $I\left(x_{0}\right) \cap A^{0}$ is both open and closed in $I\left(x_{0}\right)$, a connected set. Thus either $I\left(x_{0}\right) \cap A^{0}$ is void or $I\left(x_{0}\right) \cap A^{0}$ $=I\left(x_{0}\right)$. If $I\left(x_{0}\right) \cap A^{0}$ is void, since $J\left(x_{0}\right)=I\left(x_{0}\right)^{*}$, we have $J\left(x_{0}\right)$ $\subseteq C \backslash A^{0}$, a contradiction by condition 3 . Hence the zero for $C$ belongs to $I\left(x_{0}\right)$ which is contained in $A^{0}$ and the lemma is proved. 
Corollary. Let $C$ be a continuum that is a semigroup with zero. Let $A=A^{*}$ be a subset of $C$ such that: (1) $A^{*} \backslash A^{0}$ is a nontrivial group with identity $u$; and (2) $A^{0}$ is nonvoid and contained in $J(u)$. Then the zero for $C$ belongs to $A^{0}$.

Proof. This follows immediately from the above lemma letting $x_{0}=u$.

Lemma 6. Let $S$ be as defined above. The zero of $S$ belongs to $(\tau[0, \infty))^{*}$.

Proof. Let $(\tau[0, \infty))^{*}$ be denoted by $T$ and assume that $0 \notin T$. First let us note that if $f$ is any other idempotent in $T, f$ belongs to $B$ and therefore $e=e f=f e=f$ since $T$ is commutative. It follows from this fact that $T$ is a group since it contains at most one idempotent which acts as an identity.

(i) To show $T$ is one-dimensional. If $T$ were two-dimensional, then $T$ would contain a two-cell, [1, p. 239], and would, therefore, be both opened and closed in $S$ which is a contradiction. Hence $T$ is a onedimensional group.

(ii) To show $T$ is a circle. First let us note that for any $x \neq 0$ in $S^{0}$ there exists $f \in B$ such that $x=x f=f x$. This follows from the fact that for any sequence $s(x)$ of $2^{n}$ th roots of $x$ there is an $f$ in $Z(s(x))$ which acts as an identity for $s(x)$. From this fact it follows that $e$ is a right unit for $S$, since for any $x$ in $S, x e=(x f) e=x(f e)=x f=x$, where $f$ is as above. Thus $T$ can be considered as a compact group acting on $S$. Hence $T$ is locally connected, since $T$ is a one-dimensional orbit $[1$, p. 248]. Thus $T$ is a circle group, since the only connected, onedimensional groups are the circle group and the solenoid, and the latter is not locally connected.

(iii) Since $T$ is a circle, $S \backslash T=P \cup Q$, where $P$ and $Q$ are open, disjoint, nonempty sets. $P^{*} \backslash P^{0}=T=Q^{*} \backslash Q^{0}$, hence $P^{*}$ and $Q^{*}$ satisfy the hypothesis for the corollary of Lemma 5, since $P \subseteq J(e)$ and $Q \subseteq J(e)$. Thus $0 \in P \cap Q$, which is a contradiction. From this last contradiction it follows that 0 belongs to $T$.

Lemma 7. For all real $t, 0<t<\infty, \lim \tau(t)^{n}=0$.

Proof. In the following $\Gamma(a)=\left\{a, a^{2}, a^{3}, \cdots\right\} *$. Since the only idempotents in $T$ are 0 and $e$, there exists at least one $t_{0}$ such that $0 \in \Gamma\left(\tau\left(t_{0}\right)\right)$ for otherwise $T$ would be a group which is a contradiction. Now for $a>t_{0}, a=t_{0}+b$ for some real $b$, hence $\tau(a)=\tau\left(t_{0}\right) \tau(b)$. Thus $\tau(a)^{n}=\tau\left(t_{0}\right)^{n} \tau(b)^{n}$ for all positive integers $n$. Since $0 \in \Gamma\left(\tau\left(t_{0}\right)\right)$, $\lim \tau\left(t_{0}\right)^{n}=0$. Now either $e \in \Gamma(\tau(b))$ or $0 \in \Gamma(\tau(b))$, but in either case, there is a subsequence $(\tau(b))_{j}^{n}$; converging to an idempotent. From this it follows that $\lim \tau(a)^{n_{i}}=\lim \tau\left(t_{0}\right)^{n_{j}} \lim \tau(b)^{n_{j}}=0 \lim \tau(b)^{n_{j}}=0$. 
Therefore $\lim \tau(a)^{n}=0$ for all real $a>t_{0}$. For $0<b<t_{0}$, there exists an integer $n$ such that $t_{0} \leqq n b$, or $t_{0} / n \leqq b$. Since $\tau\left(t_{0} / n\right)^{n}=\tau\left(n t_{0} / n\right)=\tau\left(t_{0}\right)$, we have $\Gamma\left(\tau\left(t_{0}\right)\right) \subseteq \Gamma\left(\tau\left(t_{0} / n\right)\right)$ and therefore $0 \in \Gamma\left(\tau\left(t_{0} / n\right)\right)$. Since $t_{0} / n \leqq b$, the preceding argument applies, therefore $\lim \tau(b)^{n}=0$. Hence the lemma is true.

Lemma 8. $T=\tau[0, \infty) \cup 0$.

Proof. The proof of this lemma follows immediately from the proof in $[3$, p. 18] for a more general situation.

LEMmA 9. $T$ is an I-semigroup and there exists $t_{0}$, either finite or equal to $\infty$ such that $T=\tau\left[0, t_{0}\right) *$ and $\tau$ is one-one on $\left[0, t_{0}\right)$.

Proof. Clearly $T$ is an arc with endpoints $e$ and 0 . Since $e$ acts as an identity for $T$ and 0 functions as a zero, $T$ is an $I$-semigroup. If there exists a finite $t_{0}>0$ such that $\tau(t)=0$, let $t_{0}=\inf \{t: \tau(t)=0\}$. If there exists no $t$ such that $\tau(t)=0$, let $t_{0}=\infty$.

If $\tau$ is not one-one on $\left[0, t_{0}\right)$, there must exist real numbers $r$ and $s$ in $\left[0, t_{0}\right)$ such that $r<s$ and $\tau(r)=\tau(s)$. Under this assumption, by a straightforward argument, making use of the fact that for any positive integer $n, \tau(n s-(n-1) r)=\tau(r)$, it can be shown that $T \subseteq \tau[0, s]$, a contradiction since $s<t_{0}$.

Lemma 10. For $s$ in $S, s=f j$ where $f \in B$ and $j \in T$. Moreover, if $f j=h k$ for some $f, h \in B$ and $j, k \in T$, then $j=k$.

Proof. Let $\theta: B \times T \rightarrow S$ be defined by $\theta(g, t)=g t$. If $\theta$ is not onto there exists $p$ in $S$ such that $p \notin \theta(B \times T)$. Let $\delta: S \backslash p \rightarrow B$ be such that $\delta$ is continuous and for $x$ in $B, \delta(x)=x$. Now define $\alpha: B \times T \rightarrow B$ by $\alpha(f, t)=\delta(\theta(f, t))$. Since $T$ is compact and connected it follows that the identity function on $B$ is homotopic to a constant function. For $\alpha(x, e)=\delta(\theta(x, e))=\delta(x e)=\delta(x)=x$ for any $x$ in $B$, and $\alpha(x, 0)$ $=\delta(0)=h_{0}$ for some $h_{0}$ in $B$. Hence $i$, the identity on $B$ and $g$ the function defined by $g(x)=h_{0}$ for $x$ in $B$ are homotopic, a contradiction. Thus $\theta$ is onto and the first part of the lemma is true.

If $f j=h k$ for $f$ and $h$ in $B, j$ and $k$ in $T$, then $e(f j)=e(h k)$. Since $e$ is the identity for $T$, it follows that $j=e j=(e f) j=e(f j)=e(h k)=(e h) k$ $=e k=k$.

Lemma 11. For $f$ and $h$ in $B, j$ and $k$ in $T,(f j)(h k)=f(j k)$.

Proof. This follows easily from the fact that $e$ is the identity for $T$ and $e g=e$ for any $g$ in $B$. For $(f j)(h k)=f(j e)(h k)=(f j)(e h) k$ $=(f j)(e k)=f(j k)$.

With this lemma the proof of Theorem A is concluded. 
Examples. (1) Let $S$ be the two-cell and define $m(x, y)=x|y|$, where $m$ is the multiplication on $S$ and the right side of the equality denotes multiplication of $x$ by the absolute value of $y$ in the ordinary way. In this example, the representation of $x=f t$, for $f$ in $B$ and $t$ in $T$ is unique. The following example shows that this need not always be true.

(2) Let $[e, f], e \neq f$, be a line segment with multiplication for $x$ and $y$ in $[e, f]$ defined by $x y=x$. Let $L=[e, f] \times[0,1]$, and for $(x, t)$ and $(y, s)$ let the product $(x, t)(y, s)=(x, t s)$ where the product $t s$ is real multiplication in the unit interval.

In $L[e, f] \times 0$ is a closed ideal, so denote by $Q$ the Rees quotient of $L$ with this ideal and let $\pi$ be the natural map from $L$ onto $Q$. From [7] $Q$ is a semigroup. Choose $a$ in $(0,1)$ and consider $a$ fixed in the following discussion. Define $\theta: Q \rightarrow Q$ by

$$
\theta(\pi(h, t))=\left\{\begin{array}{l}
\pi(e, t) \text { for } h=e \text { or } h=f \text { and } t \in[0, a] \\
\pi(h, t) \text { otherwise. }
\end{array}\right.
$$

Let $Q_{0}=\theta(Q)$. By defining multiplication in $Q_{0}$ in the obvious way, $Q_{0}$ is a semigroup.

Now let $L_{1}=\left[e_{1}, f_{1}\right] \times[0,1]$ be another semigroup as defined above, assuming for $x$ and $y$ in $\left[e_{1}, f_{1}\right], x y=x$. The set $\left[e_{1}, f_{1}\right] \times[0, a]$ is a closed ideal of $L_{1}$. Denote by $P$ the Rees quotient of $L_{1}$ with this closed ideal and let $\pi_{1}$ be the natural map from $L_{1}$ to $P$.

Define $\delta: P \cup Q_{0} \rightarrow P \cup Q_{0}$ in the following way:

$$
\delta(s)=\left\{\begin{array}{l}
\pi(e, t) \text { for } s=\pi_{1}\left(e_{1}, t\right) \text { where } t \in(a, 1], \\
\pi(f, t) \text { for } s=\pi_{1}\left(f_{1}, t\right) \text { where } t \in(a, 1], \\
\pi(e, a) \text { for } s=\pi_{1}\left(e_{1}, t\right) \text { where } t \in[0, a], \\
\pi(e, a) \text { for } s=\pi_{1}\left(f_{1}, t\right) \text { where } t \in[0, a], \\
s \text { otherwise. }
\end{array}\right.
$$

Let $S=\delta\left(P \cup Q_{0}\right)$. Clearly $S$ is topologically the two-cell. To define multiplication in $S$ it is necessary to consider only an element from $\delta\left(P \backslash \pi_{1}(h \times[0,1])\right)$, where $h=e_{1}$ or $f_{1}$, and an element from $Q_{0}$. This is true since any pair of elements from other sets in $S$ already have their product defined. For $p=\pi_{1}(h, t)$ for some $h \in\left(e_{1}, f_{1}\right)$ and $s=\theta(\pi(g, r))$ for $g \in[e, f], r$ and $t$ in $[0,1]$, let $s p=\pi(g, r t)$ and $p s=\pi_{1}(h, r t)$. It is easily seen that multiplication defined in this way is continuous, associative and that for $x$ and $y$ in the boundary $x y=x$.

For the particular element $p=\pi(e, a), p$ can be written as 
$p=(\pi(e, 1))(\pi(e, a))$ and also as $p=(\pi(f, 1))(\pi(e, a))$ and the idempotents $\pi(e, 1)$ and $\pi(f, 1)$ are distinct elements. In fact, for any idempotent in $\left[e_{1}, f_{1}\right], p=\left(\pi_{1}(h, 1)\right)(\pi(e, a))$.

\section{REFERENCES}

1. D. Montgomery and L. Zippin, Topological transformation groups, New York, Interscience, 1955.

2. J. L. Kelly, General topology, Princeton, D. Van Nostrand, 1955.

3. R. J. Koch, On topological semigroups, Tulane University dissertation, 1953.

4. R. J. Koch and A. D. Wallace, Admissibility of semigroups on continua, Trans. Amer. Math. Soc. vol. 88 (1958) pp. 277-287.

5. P. S. Mostert and A. L. Shields, One-parameter semigroups in a semigroup, to appear.

6. - On the structure of semigroups on a compact manifold with boundary, Ann. of Math. vol. 65 (1957) pp. 117-143.

7. D. Rees, On semigroups, Proc. Cambridge Philos. Soc. vol. 36 (1940) pp. 387400.

8. A. D. Wallace, Ideals in compact connected semigroups, Nederl. Akad. Wetensch. Proc. Series A vol. 59 and Indag. Math. vol. 18 (1956) pp. 535-539.

9. - The structure of topological semigroups, Bull. Amer. Math. Soc. vol. 61 (1955) pp. 95-112.

TUlane University 